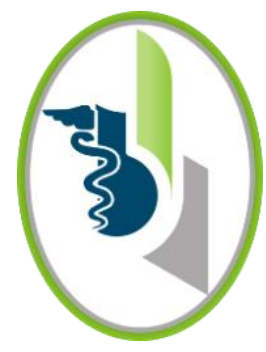

ACCESo 2 ABierto

Para citaciones: Manotas, M., Suarez, A., García, M. (2020). Determinación del riesgo de daño hepático relacionado a los medicamentos AINEs en pacientes con Chikun-Gunya y Zika. Revista Ciencias Biomédicas, 9(1), 35-43.

Recibido: 14 de septiembre de 2019 Aprobado: 18 de noviembre de 2019

Autor de correspondencia: María Cecilia García-Espiñeira mgarciae@unicartagena.edu.co

Editor: Inés Benedetti. Universidad de Cartagena-Colombia.

Copyright: (C) 2020. Manotas, M., Suarez, A., García, M. Este es un artículo de acceso abierto, distribuido bajo los términos de la licencia https://creativecommons.org/licenses/by-nc-

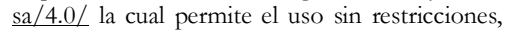
distribución y reproducción en cualquier medio, siempre y cuando el original, el autor y la fuente sean acreditados.

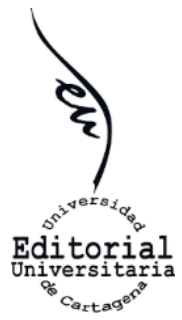

\section{Determinación del riesgo de daño hepático relacionado a los medicamentos AINEs en pacientes con Chikun-Gunya y Zika}

\author{
Determining the risk of liver damage related to NSAIDs in \\ patients with Chikun-Gunya and Zika.
}

\author{
Marco Manotas Castellar ${ }^{1}$, Amileth Suarez Causado ${ }^{2}$ iD , María Cecilia García-Espiñeira ${ }^{3}$ \\ ${ }^{1}$ Elanco animal health, Barranquilla, Colombia. \\ ${ }^{2}$ Facultad de Medicina, Universidad de Cartagena. Colombia, Colombia. \\ ${ }^{3}$ Facultad de Medicina, Universidad de Cartagena. Colombia.
}

\section{RESUMEN}

Introducción: El daño hepático inducido por fármacos es la causa más común de muerte por insuficiencia hepática aguda y representa aproximadamente el $10 \%$ de los casos en todo el mundo. Los AINEs son el grupo farmacológico con mayor número de casos de daño hepático reportados; su consumo ha aumentado debido al tratamiento de enfermedades emergentes como el virus Chikun-gunya y Zika, siendo el departamento de Bolívar uno de los principales focos de estas enfermedades.

Objetivo: Determinar el riesgo de daño hepático relacionado con los medicamentos AINES en pacientes afectados con Chikun-gunya y Zika.

Métodos: Estudio analítico observacional retrospectivo realizado en el Hospital Universitario del Caribe en el departamento de Bolívar - Colombia. Se revisaron 432 historias clínicas de pacientes hombres y mujeres con edades entre los 18-62 años que ingresaron al servicio de urgencias entre los años 2014 al 2016 y fueron diagnosticados con Chikun-gunya y Zika.

Resultados: Fueron incluidas 112 historias clínicas de las cuales el 74,1\% de los pacientes tenían diagnóstico de Chikun-gunya y tratamiento con AINES. La mediana de edad fue de 50 años (RIC: 38 - 58) y el promedio de edad fue de 48 años (DE: 14,2). Se encontraron diferencias estadísticas en el sexo, siendo más frecuente el femenino en el grupo con daño hepático encontrado en $54,3 \%$ comparado con un $7,8 \%$ en aquellos sin hepatotoxicidad, $(\mathrm{p}<0,0001)$.

Conclusiones: La variable más asociada a la hepatotoxicidad en pacientes con Chikun-gunya y tratamiento con AINES fue el sexo femenino, lo que indica un mayor riesgo de desarrollo de dicha entidad.

Palabras Clave: Chikun-gunya; Zika; AINES; hepatotoxicidad.

\section{ABSTRACT}

Introduction: Drug-induced liver damage is the most common cause of death due to acute liver failure and accounts for about $10 \%$ of cases worldwide. The NSAIDs are the pharmacological group with the highest number of reported liver damage cases, its consumption has increased due to the treatment of emerging diseases such 
as Chikun-gunya and Zika virus, being the department of Bolívar one of the main foci of these diseases.

Objective: to determine the risk of liver damage related to NSAID drugs in patients affected with Chikun-gunya and Zika.

Methods: Retrospective temporal observational analytical study carried out at the University Hospital of the Caribbean, department of Bolívar - Colombia. The medical records of male and female patients between the ages of 18-62 who entered the emergency room between 2014 and 2016 and were diagnosed with Chikungunya and Zika were reviewed.

Results: $74.1 \%$ of patients diagnosed with Chikun-gunya were treated with NSAIDs. The median age was 50 years (IQR: $38-58$ ), the average was 48 years (SD: 14.2). Statistical differences were found in sex, females being more frequent in the group with liver damage found in $54.3 \%$ compared with $7.8 \%$ in those without hepatotoxicity, $\mathrm{p}<0.0001$.

Conclusion: The variable associated with hepatotoxicity in patients with Chikungunya and treatment with NSAIDs were female sex, which indicates an increased risk of development of such entity.

Keywords: Chikun-gunya; Zika; NSAID; hepatotoxicity.

\section{INTRODUCCIÓN}

El daño hepático causado por medicamentos, drogas de abuso o remedios medicamentosos es un importante problema de salud pública que afecta a toda la población sin discriminar etnia o clase social (1). La hepatotoxicidad (HTX) se define como la lesión o daño hepático producido por la exposición a un medicamento $\mathrm{u}$ otros agentes no farmacológicos (2). Se estima que 1 de cada 10 pacientes muere o requiere trasplante hepático (3). La principal célula diana de la injuria es el hepatocito, siendo la hepatitis aguda la forma más frecuente de la hepatotoxicidad (90\% de los casos) (4) cualquier célula parenquimatosa o no parenquimatosa del hígado puede resultar dañada de forma aislada o combinada y el tipo de lesión va a depender fundamentalmente de la célula hepática predominantemente afectada $(5,6)$.

Entre los principales agentes farmacológicos que pueden producir riesgo de hepatotoxicidad son los Antiinflamatorios No Esteroideos (AINES), grupo farmacológico que con mayor frecuencia se prescribe en la práctica clínica diaria para el tratamiento y manejo de diferentes entidades. Los cuales han sido vinculados con la (HTX) con una incidencia aproximada de 1 a 10 por 100.000 exposiciones al año (2). Los AINES son metabolizados en el hígado, proceso que inicia en el retículo endoplásmico liso de los hepatocitos por la acción de enzimas citocromo $\mathrm{P} 450$, pudiéndose originar metabolitos reactivos con capacidad de causar daño en el hígado o en otros órganos (7).

Los AINES se encuentran entre los medicamentos más prescritos en todo el mundo, esta clase heterogénea de fármacos incluye la aspirina, el acetaminofén y otros agentes inhibidores de la ciclooxigenasa (COX), selectivos o no (8). El acetaminofén considerado como un derivado de los AINES es uno de los fármacos que con mayor frecuencia es imputado como causa de HTX en el Reino Unido y en Estados Unidos, esto se debe a que el metabolismo hepático del fármaco que a través de la vía oxidativa del citocromo $\mathrm{P} 450$, produce un metabolito reactivo altamente tóxico, $\mathrm{N}$ acetil-paraminobenzoquinonaimina (NAPBQI) (9). En un estudio prospectivo multicéntrico del grupo cooperativo Acute Liver Failure Study Group 
(ALFSG), que valora los ingresos consecutivos de pacientes con falla hepática aguada agudo en 17 hospitales de Estados Unidos, señala los medicamentos (incluyendo los casos de intoxicación por paracetamol: $39 \%$ ) como la causa más frecuente de esta enfermedad, superando incluso a las producidas por los virus de hepatitis A y B (10).

En Colombia el consumo de AINES es elevado, se estima que hasta el año 2013 existían en el Instituto de Vigilancia de Medicamento y Alimentos (INVIMA) 1.043 registros sanitarios correspondientes al grupo de los AINES, ocho de los principios activos de este grupo farmacológico poseen doble condición de venta (venta sin prescripción facultativa o libre y venta con fórmula médica) y alcanzan el 70\% de las autorizaciones de comercialización de AINES en Colombia (11). Lo cual supone un problema ya que la condición de venta libre de los medicamentos tiene relación directa con la automedicación, entendida esta como la selección y el uso de medicamentos, sin prescripción médica (12).

El consumo de AINES Se vio aumentado en el país, por ser el tratamiento de elección para enfermedades emergentes como el Chikun-gunya y Zika presentadas durante el periodo de 2014 a 2016 (13). Según el Ministerio de Salud y Protección Social, hasta el 20 de diciembre del 2014 se habían presentado en Colombia 74.566 casos confirmados del virus, y los departamentos con mayor número de personas afectadas eran Norte de Santander, Bolívar, Sucre, Atlántico, Guajira y Cesar.

Los síntomas de enfermedad en humanos infectados por el mosquito aparecen generalmente después de un período de incubación de 3 a 7 días (rango de 1-12 días) (14), clínicamente se manifiesta por fiebre, asociada a dolor articular severo (15). La artralgia es típicamente simétrica, y afecta principalmente las articulaciones periféricas como las muñecas, rodillas y tobillos (11), el tratamiento aprobado en Colombia según el ministerio de salud fue el manejo con acetaminofén $(15,16)$.
Al finalizar la aparición del Chikun-gunya en Colombia, se produjo la llegada de un segundo arbovirus, el virus Zika, del cual se reportaba un brote epidémico en Brasil (17), y con las condiciones epidemiológicas idóneas en Colombia se inició la transmisión autóctona y con ello una epidemia que desde septiembre de 2015 (semana epidemiológica 40) hasta el 23 de enero de 2016 (semana epidemiológica 3) había producido 20.297 casos, 1.050 confirmados por laboratorio procedentes de 30 entidades territoriales, 17.115 casos confirmados por clínica procedentes de 30 entidades territoriales y 2.132 casos sospechosos procedentes de 33 entidades territoriales (18).

Al ser los AINES los principales fármacos para el manejo de los síntomas que causan estos virus, y debido al aumento en el consumo de estos se hace necesario investigar las secuelas de daño hepático por AINES en enfermedades emergentes como Chikun-gunya y Zika, entre los años 2014 al 2016 en Cartagena, Colombia.

Se ha demostrado a nivel mundial que los estudios de hepatoxicidad han generado un mayor control de los medicamentos y en algunos casos retiro del mercado a los cuales se les ha demostrado su potencial hepatotóxico como es el caso del sulindaco y la nimesulide, este último comercializado en Colombia, donde no se encuentran estudios que informen acerca de esta problemática. El objetivo de este estudio fue determinar el riesgo de hepatotoxicidad relacionado al consumo de medicamentos AINES en pacientes afectados con Chikun-gunya y Zika.

\section{MÉTODOS}

Estudio analítico observacional retrospectivo realizado en el Hospital Universitario del Caribe, Cartagena, Colombia, con el fin de identificar el riesgo de hepatotoxicidad asociada al consumo de AINES en pacientes con Chikun-gunya y Zika. Para lo que se hizo una revisión de las historias clínicas individuales de 432 pacientes atendidos entre los años 2014 al 2016 que fueron diagnosticados con dichas patologías, Con edades entre 18 años 62 años. 
Fueron excluidas aquellas historias clínicas de pacientes que presentaban deficiencia de alfa-1 antitripsina, absceso hepático amebiano, hepatitis autoinmunitaria, atresia biliar, cirrosis, coccidioidomicosis, colestasis medicamentosa, hígado graso, hematocromatosis, hepatitis A, B, C y $\mathrm{D}$, carcinoma hepatocelular carcinoma hepatocelular, cirrosis biliar primaria, absceso hepático piógeno, síndrome de Reye, colangitis esclerosante y enfermedad de Wilson.

En este estudio se estableció que debe producirse al menos una de las siguientes alteraciones de la bioquímica para ser considerado una lesión hepática: a) elevación de la ALT (alanina aminotransferasa) dos veces por encima del límite alto normal (LAN), b) aumento de la concentración de bilirrubina conjugada dos veces por encima del LAN y c) elevación de la AST (aspartato aminotransferasa), de la FA (fosfatasa alcalina) y de la concentración total de bilirrubina, siempre que uno de los valores sea el doble del LAN (19).Se utilizó la metodología de CIOMS/ RUCAM como método de evaluación de la causalidad, esta escala evalúa siete parámetros los cuales son: tiempo transcurrido hasta la aparición de la lesión tras el inicio del fármaco, evolución posterior de la lesión después de suspender el medicamento, factores de riesgo específicos (edad, consumo de alcohol, embarazo), uso de otros medicamentos con potencial de daño hepático, exclusión de otras causas de enfermedad hepática, potencial conocido de hepatotoxicidad del fármaco implicado y respuesta a la re-exposición. Las puntuaciones totales varían de menos de 0 a 14 con puntuaciones de 3 o menos que indican hepatotoxicidad improbable, $4-5$ posible, 8 probable $\mathrm{y}>8$ altamente probable (20).

El análisis estadístico descriptivo se realizó utilizando variables cualitativas mediante el cálculo de frecuencias absolutas y relativas, por su parte las cuantitativas se expresarán con medidas de tendencia central tipo mediana $(\mathrm{Me})$ y promedio $(\mathrm{X})$ con sus respectivas medidas de dispersión rango inter cuartílico (RIC) y desviación estándar (DE). Como prueba de hipótesis para comparar entre personas con y sin daño hepático se utilizará la prueba de Mann Whitney para edad, mientras que en las variables cualitativas se usará la prueba Chi2 o el test Exacto de Fisher según se necesario, un valor de $\mathrm{p}<0,05$ será considerado como estadísticamente significativo. Finalmente se estimarán odds Ratio (OR) crudos para asociar sexo, IMC, diagnóstico de Chikun-gunya y tratamiento con AINES como factores determinantes de daño hepático, en este último se calcularán demás sus respectivos intervalos de confianza al $95 \%$ (IC $95 \%$ ).

Esta investigación acoge y respeta los principios rectores de la investigación en humanos; enunciados en la declaración de Helsinki. Por ser un estudio descriptivo de serie de casos, esta es una investigación de riesgo mínimo para los participantes, de acuerdo a la resolución 008430 de 1993 del Ministerio de Salud de Colombia en su Artículo 11.

La confidencialidad de los datos obtenidos se garantizó utilizando a manera de identificación el número de la historia clínica. Se limitó al acceso de los instrumentos de investigación únicamente a los investigadores principales (Artículo 8 de la resolución 008430 de 1993 del Ministerio de Salud) (21).

\section{RESULTADOS}

De las 432 historias clínicas revisadas fueron incluidos en el estudio 112 pacientes de los cuales el $74,1 \%(\mathrm{n}=82)$ tenían diagnóstico de Chikun-gunya y tratamiento con AINES, el porcentaje restante no tenía las anteriores condiciones, ninguno tuvo diagnóstico de Zika. La mediana de edad fue de 50 años (RIC: 38 - 58), el promedio fue de 48 años (DE: $14,2)$, el 77,7 \% $(n=87)$ de los pacientes fueron de sexo masculino. La procedencia fue urbana en un 50 $\%(n=56)$ y el estrato socioeconómico más frecuente fue el 1 con 94,6\% $(n=106)$. El grado de escolaridad más alto alcanzado referido por los pacientes fue primaria con $92,0 \%(n=103)$ (Tabla 1$)$. 
Tabla 1. Características sociodemográficas de los pacientes incluidos

\begin{tabular}{lcc}
\hline & $\mathbf{n}$ & $\mathbf{\%}$ \\
\hline Edad Me (RIC) $\overline{\mathrm{X}} \pm \mathrm{DE}$ & $50(38-58)$ & $48 \pm 14,2$ \\
Sexo & 25 & \\
$\quad$ Femenino & 87 & 22.3 \\
$\quad$ Masculino & & 77.7 \\
Residencia & 56 & 50.0 \\
$\quad$ Rural & 56 & 50.0 \\
$\quad$ Urbano & & \\
Estrato Socioeconómico & 106 & 94.6 \\
1 & 5 & 4.5 \\
2 & 1 & 0.9 \\
3 & & \\
Grado de escolaridad & 4 & 3.6 \\
Ninguno & 103 & 92,0 \\
Primaria & 2 & 1.8 \\
Primaria incompleta & 3 & 2.7 \\
$\quad$ Secundaria I & & \\
\hline
\end{tabular}

El índice de masa corporal mostró a un $87,5 \%$ $(\mathrm{n}=98)$ de los participantes en infrapeso, 8,9\% $(n=10)$ en sobrepeso y $3,6 \%(n=4)$ en obesidad. El daño hepático o hepatotoxicidad se observó en el $68,8 \%$ de la muestra correspondiente a 77 pacientes. Al comparar la edad, el sexo, Índice de Masa Corporal (IMC), diagnóstico de Chikun-gunya y tratamiento con AINES se encontraron diferencias estadísticas en el sexo siendo más frecuente el femenino en el grupo con daño hepático encontrado en $54,3 \%(n=17)$ comparado con un $7,8 \%(n=6)$ en aquellos sin hepatotoxicidad, $\mathrm{p}<0,0001$.

Tabla 2. Comparación y asociación entre edad, sexo, índice de masa corporal, diagnóstico de Chikun-gunya y tratamiento con AINES como factores determinantes de hepatotoxicidad

\begin{tabular}{|c|c|c|c|c|c|c|}
\hline & $\begin{array}{c}\text { Total } \\
\text { participantes }\end{array}$ & $\begin{array}{c}\text { Con daño } \\
\text { hepático } \\
\mathbf{n}=\mathbf{3 5}\end{array}$ & $\begin{array}{c}\begin{array}{c}\text { Sin daño } \\
\text { hepático } \\
\mathbf{n}=77\end{array} \\
\end{array}$ & Valor $\mathbf{p}$ & OR & IC $95 \%$ \\
\hline Edad Me (RIC) & $50(38-58)$ & $51(32-65)$ & $50(41-65)$ & 0,4567 & NA & \\
\hline \multicolumn{7}{|l|}{ Sexo } \\
\hline Femenino & $25(22,3)$ & $19(54,3)$ & $6(7,8)$ & $<0,0001$ & 14,05 & $4,83-40,80$ \\
\hline Masculino & $87(77,7)$ & $16(45,7)$ & $71(92,2)$ & & 0,071 & $0,024-0,2067$ \\
\hline \multicolumn{7}{|l|}{ IMC } \\
\hline Menor a 18.5 & $98(87.5)$ & $34(97,1)$ & $64(83,1)$ & 0,0604 & 4,78 & $0,58-39,33$ \\
\hline De $25-29.9$ & $10(8.9)$ & $1(2,9)$ & $9(11,7)$ & 0,1679 & 0,22 & $0,02-1,82$ \\
\hline \multicolumn{7}{|l|}{$\begin{array}{l}\text { Mayor a } 30 \\
\text { Chikun-gunya }\end{array}$} \\
\hline $\begin{array}{l}\text { Chikun-gunya } \\
\mathrm{Si} \\
\text { No }\end{array}$ & $\begin{array}{l}83(74.1) \\
29(25.9)\end{array}$ & $\begin{array}{c}6(17,1) \\
29(82.9)\end{array}$ & $\begin{array}{c}77(100,0) \\
0(0,0)\end{array}$ & $<0,0001$ & 0.00 & $\mathrm{NC}$ \\
\hline \multicolumn{7}{|l|}{$\begin{array}{l}\text { Tratamiento con } \\
\text { AINES }\end{array}$} \\
\hline $\begin{array}{l}\mathrm{Si} \\
\mathrm{No}\end{array}$ & $\begin{array}{l}83(74.1) \\
29(25.9) \\
\end{array}$ & $\begin{array}{r}6(17,1) \\
29(82,9) \\
\end{array}$ & $\begin{array}{c}77(100,0) \\
0(0,0)\end{array}$ & $<0,0001$ & 0.00 & $\mathrm{NC}$ \\
\hline
\end{tabular}

Fuente: datos del estudio 
La comparación del estado nutricional según el IMC no mostró diferencias estadísticas significativas, el diagnóstico de Chikun-gunya y tratamiento con AINES fueron más frecuentes en el grupo sin daño hepático observado en el $100 \%$ de estos sujetos, $\mathrm{p}<0,0001$. La estimación de los OR solo fue posible para la variable sexo observando para el sexo femenino un $\mathrm{OR}=14,05$ (IC $95 \%$ 4,83 - 40,80), para el masculino $\mathrm{OR}=0,071$ (IC $95 \%$ : 0,024 - 0,2067) y en las variables diagnóstico de Chikun-gunya y tratamiento con AINES OR $=0,00$ con intervalos de confianza no calculables por incluir un valor 0 dentro de su cálculo (Tabla 2).

\section{DISCUSIÓN}

La lesión hepática inducida por fármacos, se considera como la causa más importante de toxicidad hepática (20). En el presente estudio se evidenció que la hepatotoxicidad estuvo presente en el 68,8\% de los pacientes participantes en el estudio, de acuerdo con los resultados obtenidos por Cano (22), los AINES fueron el grupo de fármacos más importantes que puede causar alteraciones a nivel hepático, esto puede deberse a reacciones de tipo idiosincrásico en algunos de los pacientes, o por casos de abuso o sobredosis por el uso indiscriminado de los mismos.

Con respecto a las condiciones sociodemográficas de los pacientes participantes en el estudio, tuvieron una mediana de edad de 50 años (RIC: 38 - 58), y promedio de 48 años (DE: 14,2), considerado esta como una variable de riesgo para el desarrollo de daño hepático, se ha descrito en la literatura (23). A partir de los 40 años se incrementa el riesgo de toxicidad por fármacos, siendo la incidencia de esta es de 2-3 veces más frecuente en adultos que en el resto de la población; asociado por el uso concomitante de otros fármacos, a la existencia de comorbilidades y las diferencias en la biodisponibilidad y metabolismo de los fármacos (24).

El grado de escolaridad más alto alcanzado referido por los pacientes fue primaria completa en la mayoría de los pacientes. El nivel de escolaridad de los pacientes influye en el autocuidado que estos tengan de su propia salud, es por eso que se considera como una variable importante en el manejo y tratamiento de diversas patologías, se infiere así que las personas que con un mayor nivel educativo tienen mayores probabilidades de hacer buen uso de los fármacos, como son los AINES para el manejo de los síntomas producidos por Chikungunya y Zika $(25,26)$.

En lo que concierne a las características clínicas de los pacientes el 74,1 \% tenían diagnóstico de Chikun-gunya y tratamiento con AINES, el porcentaje restante no tenía las anteriores condiciones, ninguno tuvo diagnóstico de Zika. En la actualidad no existe aún un tratamiento específico para el tratamiento del Chikun-gunya y Zika, solo está establecido el manejo de los síntomas en los pacientes, en el caso del dolor y la fiebre el fármaco recomendado según la Organización Mundial y Panamericana de la Salud es el paracetamol $(27,28)$.

Al comparar el sexo con el diagnóstico de Chikungunya y tratamiento con AINES se encontraron diferencias estadísticas en el sexo siendo más frecuente el femenino en el grupo con daño hepático encontrado con una $\mathrm{p}<0,0001$, esto guarda relación con lo expresado por García (23) en España, quien señala que entre los factores de riesgo posiblemente relacionados con el desarrollo de RAH por AINE se encuentra el pertenecer al sexo femenino. De igual forma Cano evidenció que las mujeres son más susceptibles al desarrollo de hepatoxicidad (21). Las mujeres parecen tener mayor riesgo para el desarrollo de alteraciones a nivel hepático que puede presentarse forma de hepatitis aguda o crónica, posiblemente, esto se relaciona con el menor peso medio de las mujeres, las diferencias metabólicas o con factores hormonales que predisponen a un aumento en la toxicidad (29).

En cuanto al estado nutricional según el IMC los pacientes en su mayoría estaban en infrapeso, pero un porcentaje significativo se encontraban en sobrepeso y obesidad, esta variable no mostró diferencias estadísticas significativas en el diagnóstico de Chikun-gunya y tratamiento con 
AINES, lo que difiere a lo encontrado por Diez en España donde el IMC promedio de pacientes fue de 29.8, y se asoció con la presencia de Hepatotoxicidad (30). Se ha evidenciado que las alteraciones metabólicas en los pacientes con un IMC mayor a 25 se producen alteraciones a nivel enzimático, y una mayor susceptibilidad de metamorfosis grasa que se asocia a deficiencias nutricionales, es por eso que los profesionales de la salud deben hacer énfasis en la prevención de enfermedades hepáticas y sus complicaciones, enfocándose en la promoción de los estilos de vida y sus hábitos alimenticios saludables como factor protector en la obesidad (31).

Este estudio se convierte en un instrumento para la comunidad científica, puesto que hay evidencia d la falta de programas que busquen el uso racional de fármacos, en especial los que son de venta libre como los AINES, en los cuales existe la creencia de ser inocuos como es el caso del acetaminofén, desconociendo los potenciales tóxicos que tiene la molécula cuando su consumo excede los niveles permitidos.

La principal limitación de este estudio fue la imposibilidad de estudiar otras variables clínicas en estos pacientes que nos permitiera aclarar otras posibles causas de hepatotoxicidad, como consumo de alcohol, sustancias psicoactivas, u otros fármacos en forma crónica como las estatinas, que también pueden contribuir a la aparición de esta entidad.

Se puede concluir que la hepatoxicidad estuvo presente en el $68,8 \%$ de los pacientes participantes en el estudio, en su mayoría hombres, La asociación de variables hepatotoxicidad en pacientes con Chikun-gunya y tratamiento con AINES fue mayor en el sexo femenino, lo que indica un mayor riesgo de desarrollo de dicha entidad. Se evidenció además que el 74,1 \% tenían diagnóstico de Chikun-gunya y tratamiento con AINES, el porcentaje restante no tenía las anteriores condiciones, ninguno tuvo diagnóstico de Zika, los participantes en el estudio además contaban en su mayoría con alteraciones nutricionales en su mayoría tenían un infrapeso de acuerdo con su IMC.
CONFLICTOS DE INTERESES: Los autores no declaran conflictos de intereses.

AGRADECIMIENTOS: Al Hospital Universitario del Caribe, Cartagena, Colombia

\section{REFERENCIAS}

1. Chávez E. Hepatotoxicidad por fármacos. Medwave [Internet]. 2006;(11):177-91. Disponible en:

http://www.medwave.cl/link.cgi/Medwave/Puesta Dia/APS/1977

2. Tejada F. Hepatotoxicidad por Fármacos. Rev Clin Med Fam. 2010; 3(3): 177-191. Disponible en: http://scielo.isciii.es/scielo.php?script=sci arttext \&pid=S1699-695X2010000300006\&lng=es.

3. Morales L, Vélez N, Muñoz O. Hepatotoxicidad: patrón colestásico inducido por fármacos. Revista Colombiana de Gastroenterología. 2016; 31(1): 3647

4. Rojas C, Quesada M, Esquivel N Intoxicación por acetaminofén: diagnóstico y abordaje en el adulto. Revista Médica Sinergia. 2019; 4(7): e257-e257.

5. Cano A, Posada L, Muñoz P. Toxicidad hepática causada por medicamentos: revisión estructurada. Rev Colomb Gastroenterol [Internet]. 2017; 32(4):337-48.

6. Gunawan B, Kaplowitz N. Mechanisms of DrugInduced Liver Disease. Clin Liver Dis. 2007;11(3):459-75.

7. Bruguera Cortada M. Toxicidad hepática. Med Programa Form Médica Contin Acreditado [Internet]. 2004;9(7):435-42. Disponible en: http://linkinghub.elsevier.com/retrieve/pii/S02113 $\underline{44904700557}$

8. Batlouni M. Antiinflamatorios No Esteroides: Efectos Cardiovasculares, Cerebrovasculares y Renales. Soc Bras Cardiol MCMXLIII. 2010;94(4):538-46.

9. Mühlbauer M. Paracetamol, um AINE particular. Ciência Atual-Revista Científica Multidisciplinar do Centro Universitário São José. 2016; 7(1): 2-20 
10. García M, Andrade R, Lucena M, González R, Camargo R, Fernández E, et al. Hepatotoxicidad secundaria a fármacos de uso común. Gastroenterol Hepatol [Internet]. 2005; 28(8):461-72. Disponible en: $\quad$ http://www.elsevier.es/es-revistagastroenterologia-hepatologia-14-articulohepatotoxicidad-secundaria-farmacos-uso-comun$\underline{13079002}$

11. Solano M, Garavito G. Condición de venta de analgésicos antiinflamatorios no esteroides, legalmente autorizados para su comercialización en Colombia. Estrategias de uso racional Resumen Condition of sale of non-steroidal antiinflammatory drugs Introducción. Rev Colomb Cienc Quím Farm [Internet]. 2013;42(2):145-68. Disponible en: http://search.proquest.com/openview/76860f0a454 455806963cdaf5a8b0733/1?pqorigsite $=$ gscholar \&cbl $=2035763$

12. Del Toro M, Díaz A, Barrios Z, Castillo I. Automedicación y creencias en torno a su práctica en Cartagena, Colombia. Rev Cuid [Internet]. 2017;8(1):1509. Disponible en: http://www.revistacuidarte.org/index.php/cuidarte/ article/view/367

13. Zuluaga M, Vanegas D. El virus Chikungunya en Colombia: aspectos clínicos y epidemiológicos y revisión de la literatura. Iatreia [Internet]. 2016;29(1):65-74. Disponible en: http://www.scielo.org.co/scielo.php?script=sci artt ext\&pid=S0121-07932016000100006\&lang $=p t$

14. Ministerio de Salud y Protección Social. Lineamientos para el manejo clínico de los pacientes con el virus chikungunya (chikv). Alcance. Minist Salud [Internet]. 2013;(32):3305050.

15. Instituto de evaluacion tecnológica en salud. Control y tratamiento del chikungunya. J Chem Inf Model. 2013;53(1):1689-99.

16. De Abajo F, Montero D, Madurga M, Rodriguez L. Acute and clinically relevant drug-induced liver injury: a population-based case-control study. $\mathrm{Br} \mathrm{J}$ Clin Pharmacol [Internet]. 2004;58(1):71-80. Disponible en: http://doi.wiley.com/10.1111/j.13652125.2004.02133.x
17. Rodriguez A. Situación del Zika en Colombia: experiencia de miembros de la Red Colombiana de Colaboración en Zika (RECOLZIKA). Acta Med Peru. 2016;33(1):79-81.

18. Rodríguez A, Willamil W. El reto de Zika en Colombia y América Latina: Una urgencia sanitaria internacional. Infectio [Internet]. 2016;20(2):5961. Disponible en: http://dx.doi.org/10.1016/j.infect.2016.02.001

19. Tejada F. Hepatotoxicidad por fármacos. Revista Clínica de Medicina de Familia. 2010; 3(3): 177191

20. LiverTox: Clinical and Research Information on DrugInduced Liver Injury [Internet]. Bethesda (MD): National Institute of Diabetes and Digestive and Kidney Diseases; 2012-. Causality.[Updated 2019 Nov 20].

21. Ministerio de salud. Resolución 008430 por la cual se establece las normas académicas, técnicas y administrativas para la investigación en salud. Bogotá DC: Ministerio de salud;1993

22. Cano A, Cifuentes L, Amariles P. Toxicidad hepática causada por medicamentos: revisión estructurada. Revista Colombiana de Gastroenterología. 2017;32(4):337-48.

23. García M, Andrade R, Lucena M, González-Grande $\mathrm{R}$, Camargo R, Fernández-Bonilla $\mathrm{E}$, et al. Hepatotoxicidad secundaria a fármacos de uso común. Gastroenterología y hepatología. 2005;28(8):461-72.

24. Bonkovsky HL. Hepatotoxicity associated with supplements containing Chinese green tea (Camellia sinensis). Annals of internal medicine. 2006;144(1):68.

25. Angarita V, Marina O. Agencia de autocuidado en hipertensos usuarios de un hospital universitario en Cúcuta (Colombia). Revista Científica Salud Uninorte. 2014;30(2).

26. Morazán AFD, Batchvaroff $\mathrm{MB}$, González $\mathrm{CH}$, Andrade DSB. Conocimientos, actitudes y prácticas sobre las arbovirosis. Archivos de medicina. 2017;13(1):5. 
27. Cortes S, Vidal D, Fuentes D, Moreno A, Velázquez $\mathrm{M}$. Infección por ZIKA en mujeres gestantes en un Hospital de Chiapas, México. Revista Cubana de Investigaciones Biomédicas. 2018;37(3):1-9.

28. Organización Mundial De la Salud. Nota descriptiva Chikungunya. OMS, Washington Dc; 2017. Disponible en: https://www.who.int/es/newsroom/fact-sheets/detail/chikungunya

29. Rodríguez RM, Corraliza ES, Encinas IP, Martín AF. Factores de riesgo y mecanismos de toxicidad hepática. Medicine-Programa de Formación Médica Continuada Acreditado. 2008;10(10):6118 .

30. Diez J, Comas A. Asymptomatic hypertransaminasemia in patients in primary care. Revista espanola de enfermedades digestivas: organo oficial de la Sociedad Espanola de Patologia Digestiva. 2011;103(10):530-5.

31. Cacciola I, Scoglio R, Alibrandi A, Squadrito G, Raimondo G, Group S-MHS. Evaluation of liver enzyme levels and identification of asymptomatic liver disease patients in primary care. Internal and emergency medicine. 2017;12(2):181-6. 